\title{
EL CONSUMO DE ARTES ESCÉNICAS EN MEDELLÍN*
}

Yadira Gómez Hernández
Andrey Ramos Ramírez
Nora Espinal Monsalve

* DOI: https://doi.org/10.18601/01245996.v22n42.12. Recepción: 19-092018, modificación final: 24-09-2019, aceptación: 11-10-2019. Sugerencia de citación: Gómez, H. Y., Ramos, R. A. y Espinal, M. N. (2020). E1 consumo de artes escénicas en Medellín. Revista de Economía Institucional, 22(42), 297-323.

a Magister en Economía. Integrante del Grupo de Investigación "Economía, cultura y políticas”, Universidad Nacional de Colombia, Medellín, [lygomezh@unal.edu.co], [https://orcid.org/0000-0002-1791-5886].

b Magíster en Ciencias Económicas. Integrante del Grupo de Investigación "Economía, cultura y políticas", Universidad Nacional de Colombia, Medellín, [adramosr@unal.edu.co], [https://orcid.org/0000-0002-5795-3190].

c Doctora en Administración y Dirección de Empresas. Directora del Grupo de Investigación "Economía, cultura y políticas”, profesora asociada, Departamento de Economía, Universidad Nacional de Colombia, Medellín, [nespinal@unal.edu.co], [https://orcid.org/0000-0003-2777-7410]. 


\section{El consumo de artes escénicas en Medellín}

Resumen. En este trabajo se analizan los determinantes de la asistencia a espectáculos de artes escénicas en Medellín: teatro, danza y música. Con datos de la Encuesta de Calidad de Vida de Medellín 2014, se estiman modelos de elección discreta regularizados que permiten identificar las variables relevantes para explicar las decisiones de consumo cultural, y solucionan los problemas estadísticos asociados a la existencia de un alto número de variables explicativas. Los resultados muestran que el nivel de educación y las restricciones de tiempo son los determinantes más importantes de la asistencia a eventos de artes escénicas en la ciudad.

Palabras clave: artes escénicas, participación cultural, modelos regularizados; JEL: Z11, Z18

\section{Performing arts consumption in Medellín}

Abstract. In this paper, we analyze the determinants of attendance and the frequency of attendance of performing arts events in Medellin, specifically theater, dance, and music. Using data from the Quality of Life Survey of Medellin 2014, we estimate regularized discrete choice models that allow us to efficiently select the relevant variables that explain the cultural consumption decisions, while at the same time, solving the statistical problems that appear when the number of explanatory variables is high. Results show that the level of education and time restrictions are the principal determinants of performing arts attendance in the city.

Keywords: Performing arts, cultural participation, regularized models; JEL: Z11, Z18

\section{O consumo de artes cênicas em Medellín}

Resumo. Este artigo analisa os determinantes da participação em espetáculos de artes cênicas em Medellín: teatro, dança e música. Com os dados da Pesquisa de Qualidade de Vida de Medellín de 2014, estima-se modelos de escolha discreta regularizados que permitem a identificação de variáveis relevantes para explicar as decisões de consumo cultural e resolver os problemas estatísticos associados à existência de um elevado número de variáveis explicativas. Os resultados mostram que o nível de escolaridade e as restrições de tempo são os determinantes mais importantes da participação em eventos de artes cênicas na cidade.

Palavras-chave: artes cênicas, participação cultural, modelos regularizados; JEL: Z11, Z18 
Wuy distante de las primeras definiciones como descanso del 1 trabajo, el ocio se asocia hoy a una vida más cómoda y a diversión; se concibe como una necesidad humana cuya satisfacción es requisito de una buena calidad de vida (Cuenca, 2006). El cultivo del ocio tiene efectos motivadores, y da sentidos y significados que se pueden orientar al desarrollo personal y social. La nueva actitud social con respecto al ocio -junto con los mayores niveles de ingreso, educación y tiempo libre- ha impulsado el consumo de bienes y servicios culturales en los momentos de ocio (Frey, 1994; Yeoman, 2004), y es tema de interés en diversas disciplinas, entre ellas la economía.

Más allá de ser un mecanismo de realización del ocio, el consumo cultural tiene efectos importantes sobre el bienestar social. Se ha demostrado que tiene efectos individuales y colectivos: incrementa las habilidades cognitivas y de comprensión, mejora la salud y la autoestima, reduce y previene el crimen, aumenta la disposición a la tolerancia y la no discriminación, así como la identidad comunitaria y la cohesión social en cualquier nivel de ingresos (Pnud, 2002; VV.AA., 2004; Belfiore y Bennett, 2007). Según Horkheimer y Adorno (2009), el contacto de los individuos y comunidades con manifestaciones artísticas y culturales puede servir para transformar el espacio social y cultural, porque el arte es un medio valioso para crear comunidades sólidas en cuanto forma redes sociales entre diferentes grupos de personas y alienta el sentido de pertenencia a una comunidad.

Así, el consumo cultural tiene una afinidad electiva con las valoraciones democráticas, políticas y sociales de los consumidores, en todos los segmentos de la población (Núñez, 2016). Matarasso (1997) argumenta que el consumo de actividades culturales y artísticas promueve el desarrollo local y humano. Por su parte, Rish (2005), demuestra que la participación local y regional en actividades culturales y la diversidad étnica y económica influyen positivamente en la retención de la población y en la superación parcial de la pobreza. Otros autores, como Belfiore y Bennett (2007), también reconocen y valoran la importancia de la cultura y las artes en el desarrollo social, comunitario y económico.

Uno de los consumos culturales de mayor interés para los académicos es la asistencia a espectáculos de artes escénicas en cualquiera de sus manifestaciones: teatro, danza, música y todo espectáculo que se pueda poner en escena. $\mathrm{E} 1$ consumo de artes escénicas como actividad de ocio depende del contexto social, económico, cultural y religioso de cada época (López, 1977), y es una decisión influenciada 
por la satisfacción y el disfrute que de algún modo son un signo de distinción (Oliva y Torres, 1992).

En los trabajos de economía de la cultura se estudian tres dominios de la participación en artes escénicas: a través de los medios de comunicación, como creador y como miembro de la audiencia en eventos en vivo (McCarthy et al., 2001). Aunque la tecnología ha ampliado el alcance de las artes escénicas, se sigue asistiendo a espectáculos en vivo, y se ha demostrado que en estos espectáculos se concentran mayores desigualdades entre quienes asisten y quienes no (Borgonovi, 2004).

En las audiencias culturales del ámbito escénico existen personas con algún consumo cultural habitual, es decir, los consumidores existentes, y nuevas o potenciales: niños, jóvenes y excluidos del disfrute artístico. Para democratizar el consumo cultural, las políticas públicas deben mantener las primeras audiencias y crear las segundas, ampliar el acceso a las más vulnerables y llevar sus beneficios a toda la población. La fidelización de las nuevas audiencias así creadas requiere entender no solo los gustos, sino el papel de las experiencias satisfactorias en la frecuencia de la asistencia como motor de la demanda y la oferta de artes escénicas (Lazcano y Ladabidea, 2010).

Ante estas consideraciones teóricas y entendiendo el papel protagónico de la cultura en las transformaciones recientes de la ciudad de Medellín y la inversión reciente en estímulos a la creación y la formación de públicos, este trabajo se propone analizar los determinantes de la asistencia, y de su frecuencia, a espectáculos de teatro, danza y música en vivo, en Medellín. Con datos de la Encuesta de Calidad de Vida de Medellín 2014 (ECVM2014), se estimó un modelo de elección discreta regularizado para la decisión de asistir y un Continuation ratio model (CRM) para su frecuencia.

En la primera sección se presentan los enfoques teóricos y los principales determinantes del consumo cultural según los trabajos revisados. En la segunda se describen el caso de estudio, los datos y la metodología. En la tercera se analizan los resultados de los modelos econométricos. Por último, se formulan las conclusiones.

\section{EL CONSUMO CULTURAL}

En los estudios sobre el consumo cultural existen tres enfoques principales: el enfoque de la adicción racional (Becker, 1965; Stigler y Becker, 1977; Becker y Murphy, 1988), el enfoque sociológico (Bourdieu, 1984; Peterson, 1992) y el enfoque de aprendizaje a través del consumo (Lévy y Montmarquette, 1996). Su verificación 
empírica se ha visto favorecida por la creciente disponibilidad de información detallada y confiable sobre las decisiones de consumo de bienes y servicios culturales en contextos políticos, económicos y culturales muy diferentes. Sus resultados han ayudado a formular políticas públicas para reducir las brechas de participación cultural entre diferentes sectores de la población, lo que en el mediano y el largo plazo puede hacer posible que los beneficios de dicha participación lleguen a todos los sectores.

Basado en los supuestos de la teoría del consumidor, el primer enfoque plantea que las decisiones individuales de consumo de ciertos bienes adictivos, perjudiciales o benéficos se toman racionalmente, optimizando una función de utilidad intertemporal, con pleno conocimiento de las consecuencias del consumo presente (Seaman, 2006; Castiglione e Infante, 2016). Un bien se considera adictivo si su consumo presente aumenta con la acumulación de capital de consumo. La edad, las experiencias sucesivas y el aumento de la habilidad apreciativa adquirida en el aprendizaje por la práctica contribuyen a la acumulación de ese tipo de capital (Castiglione e Infante, 2016).

En el campo de la sociología existen dos corrientes que explican los patrones de consumo de bienes culturales. Bourdieu (1984) argumenta que el consumo de estos bienes es una expresión de la identidad de las clases sociales, de modo que hay una gran diferencia entre el consumo de las clases altas y el de las clases bajas. En contraste, $\mathrm{Pe}-$ terson (1992) encuentra evidencia empírica que sustenta la hipótesis de que los individuos de clase alta son más receptivos a diferentes formas de arte y, por tanto, son consumidores omnívoros - de bienes de la alta cultura y de bienes de la cultura popular-, lo que no ocurre en las clases bajas.

El enfoque de "aprendizaje en el consumo" de Lévy y Montmarquette (1996), en el que se centra este trabajo, plantea que cada vez que un consumidor participa en una actividad cultural obtiene un nivel de satisfacción positivo o negativo que influye en sus decisiones de consumo futuras; con base en la sorpresa que le causa cada nueva experiencia, ajusta sus preferencias revelando el gusto por la actividad cultural. Así, en el consumo actual influye más el consumo pasado que el consumo futuro (Castiglione e Infante, 2016). La idea central es que los individuos no conocen su función de utilidad, pero aprenden sus estructuras de preferencias subjetivas recurriendo a experiencias repetidas de consumo (Lévy y Montmarquette, 2003; Brito y Barros, 2005; Seaman, 2006). 
Antes de cada experiencia de consumo, el individuo desconoce la calidad del bien o servicio cultural que va a consumir, pero posee una expectativa subjetiva; la nueva experiencia le causa una sorpresa que puede ser placentera o no, y con base en ella revisa sus expectativas de consumo futuro (Lévy y Montmarquette, 2003). Como ilustración, consideremos una persona que descubre el gusto por la música barroca; aunque desconocía ese gusto, a medida que asiste a nuevas presentaciones experimenta sucesivas sorpresas positivas que lo llevan a revisar sus expectativas hacia arriba, es decir,la satisfacción que espera obtener del consumo futuro es cada vez mayor. Esta representación tiene algunas ventajas teóricas:

a. permite la heterogeneidad de gustos y la independencia entre elecciones individuales;

b. hace posible diferenciar los bienes culturales, pues cada actividad cultural ofrece nuevas posibilidades de sorpresa que podrían implicar largos periodos de aprendizaje; $y$

c. mantiene la separabilidad intertemporal de la función de utilidad condicional del consumo pasado.

Los trabajos empíricos que estudian los factores socioculturales, experienciales, simbólicos e ideológicos que llevan a asistir a espectáculos de artes escénicas en vivo han señalado, desde sus inicios, que la educación superior y el nivel de ingresos son los determinantes más significativos (Baumol y Bowen, 1966). La evidencia muestra un efecto más pronunciado de la educación; en particular, la educación en artes es el aspecto que mejor predice la asistencia a artes escénicas (Lévy y Montmarquette, 2003; Borgonovi, 2004). La importancia del nivel educativo como determinante del consumo de artes escénicas se asocia directamente con el aumento del capital cultural.

Este concepto admite dos acepciones. Desde el punto de vista de la sociología, el capital cultural alude al conocimiento, la educación, las habilidades, y ventajas de una persona que le dan mayor estatus en la sociedad (Bourdieu y Passeron, 1996). Desde el punto de vista económico es "un medio para representar la cultura [...], un activo que representa, almacena o proporciona valor cultural además de cualquier otro valor que pueda poseer" (Throsby, 2001, pp. 57 y 59). Así, es una variable stock sometida a depreciación, que genera flujos de servicios y aumenta el valor económico (Fernández y Prieto, 2009). Este activo se adquiere a través del consumo cultural, la educación general y específica en artes. Las prácticas deportivas son también una forma de capital cultural que puede favorecer el consumo cultural y el de artes escénicas. Según Stempel (2005), se puede inferir de la teoría 
de Bourdieu que las clases altas utilizan los deportes para establecer una distinción con las clases medias y bajas. Como tal, constituyen una forma de capital cultural.

Otros estudios muestran que en la demanda de artes escénicas influyen la edad, el sexo y el estado civil. La asistencia tiende a caer cuando se es adulto joven y se está consolidando una profesión y una familia (dado el mayor costo de oportunidad del tiempo) y después tiende a incrementarse. En la mayoría de los estudios se encuentra que las mujeres muestran mayor probabilidad de participar (Borgonovi, 2004; Aguado y Palma, 2012). Entre los factores que reducen la asistencia a espectáculos en vivo se encuentran las limitaciones de tiempo -que se debe repartir entre el ocio y el cumplimiento de responsabilidades- determinadas por el tipo de ocupación. También las limitaciones económicas como el nivel de ingresos y los precios, las dificultades de movilidad y el desconocimiento de los espectáculos disponibles.

En la frecuencia de la participación, además de los factores mencionados, también influye el nivel de satisfacción. Este depende de la calidad técnica, el entorno físico y la programación: el cuidado de las instalaciones donde se presentan los espectáculos, la acústica, la visibilidad de las salas. La adaptación de la programación a las preferencias de los consumidores y el cumplimiento de los horarios pueden determinar el número de veces que se asiste a espectáculos de artes escénicas en un año (Lazcano y Ladabidea, 2010).

\section{EL CASO DE MEDELLÍN}

Medellín es pionera en el país y en América Latina en la planeación de la política cultural, buscando "abordar la planeación de la ciudad desde una perspectiva cultural y presentar instrumentos que coadyuven a consolidar la acción cultural como factor estructurante y prioritario del orden social en una ciudad que se debate entre las imágenes

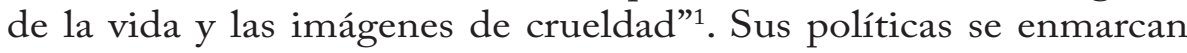
en el Plan Nacional de Cultura 2001-2010 "Hacia una ciudadanía cultural democrática”, que concibe la participación cultural como un factor de renovación, equidad social e inclusión y además, reconoce la importancia del sector cultural en el crecimiento económico y su potencial para fomentar los valores, la creatividad, la cohesión social y la búsqueda de la paz.

${ }^{1}$ Sanción del Acuerdo 41 de 1990. Alcalde Omar Flórez, 17 de septiembre de 1990. 
En 2010, después de la peor crisis de violencia desatada por el narcotráfico, se elaboró el Plan de Desarrollo Cultural de Medellín 2011-2020: "Medellín, una ciudad que se piensa y se construye desde la cultura" buscando:

\begin{abstract}
promover y consolidar a Medellín como un espacio cultural abierto al mundo, equitativo, incluyente, deliberativo y diverso que promueve la implementación de políticas culturales participativas al servicio de la consolidación de la ciudadanía cultural, del desarrollo sostenible y del mejoramiento de la calidad de vida y el bienestar de todos sus habitantes (Alcaldía de Medellín, 2011, p. 62).
\end{abstract}

Esto sitúa a la cultura como un elemento clave para mejorar la calidad de vida de los ciudadanos y transformar a Medellín, y establece un compromiso con la producción artística y la promoción del consumo cultural, que ha estado acompañado por una importante inversión en cultura en diferentes líneas y modalidades, entre ellas las artes escénicas² (Alcaldía de Medellín, 2018).

Lograr la democratización del consumo cultural, requiere políticas públicas que mantengan las audiencias existentes y estimulen la formación de nuevas audiencias. Solo así se garantizará que los beneficios individuales y sociales del consumo cultural lleguen a toda la población. En este sentido, la formulación de políticas asertivas requiere determinar con mayor precisión los factores que influyen en la asistencia a artes escénicas y en su frecuencia. Esa es la motivación de este trabajo.

\title{
METODOLOGÍA
}

\section{DATOS Y VARIABLES}

Los datos utilizados provienen de la ECVM2014, realizada por el Departamento Administrativo de Planeación de la Alcaldía de $\mathrm{Me}-$ dellín, cuya muestra se elige mediante un muestreo aleatorio simple, teniendo en cuenta la participación de cada vivienda, según estrato socioeconómico, barrio, comuna y corregimiento. Se toman los datos de las 16 comunas de la ciudad ${ }^{3}$ y se analizan las respuestas de los jefes de hogar porque su comportamiento condiciona las decisiones familiares y son los únicos entrevistados que revelan el ingreso. Considerar las respuestas de otros miembros del hogar, puede generar

${ }^{2}$ En el año 2017, \$810.000.000 fueron destinados a artes escénicas, incluyendo la financiación de la formación de públicos.

${ }^{3}$ El término "comuna" denota la unidad administrativa en que se subdivide el área urbana, que agrupa barrios o sectores. Medellín se divide en 16 comunas que agrupan 257 barrios. 
problemas de no independencia. De ese modo, la muestra utilizada se limita a 12.265 observaciones de jefes de hogar ubicados en el área urbana de Medellín en 2014. Además, partiendo de la geodatabase del Plan de Ordenamiento Territorial de Medellín 2014 (POT2014), se construyeron datos del número de equipamientos culturales y de cercanía a estaciones del $\mathrm{SITVA}^{4}$ en cada comuna.

La ECVM2014 indaga sobre asistencia a presentaciones de música, teatro y danza, y sobre la frecuencia de participación de quienes responden positivamente. Con estos datos se construyen dos variables dependientes de interés para el estudio: la asistencia a espectáculos de artes escénicas, una variable dicotómica que responde a la pregunta “¿Participa o asiste a presentaciones de artes escénicas?” y la frecuencia de asistencia, una variable categórica ordenada que clasifica a los entrevistados considerando la intensidad de la participación, como asistentes ocasionales (participan esporádicamente), asistentes frecuentes (participan mensualmente) y asistentes asiduos (participan una o más veces a la semana).

Siguiendo a Borgonovi (2004) y a Ateca (2008), en ambos casos, el modelo que relaciona las variables dependientes con las variables explicativas está dado por:

$C_{i}=f\left(\alpha+\delta S_{i}+\gamma K_{i}+\tau T_{i}+\tau U_{i}+\varepsilon_{i}\right)$

donde $S i$ son las características socioeconómicas (edad, sexo, minoría étnica, estado civil, ingreso y estrato socioeconómico), $K i$ las variables que indican el nivel de capital cultural acumulado (nivel de formación, estudios en artes, número de equipamientos culturales en el barrio y asistencia a actividades deportivas), $T i$ las restricciones de tiempo (ocupación, número de personas en el hogar) y $U_{i}$ la comuna de residencia. Las variables explicativas se seleccionan conforme a la literatura y la disponibilidad de información en la ECVM2014.

\section{ELECCIÓN DEL MODELO ECONOMÉTRICO}

La elección del modelo para analizar los perfiles de los asistentes a artes escénicas y la frecuencia de asistencia se fundamenta en el tipo de variable dependiente. Siguiendo a Cameron y Trivedi (2005), para la participación, la probabilidad de asistencia condicionada por el vector de variables independientes se define como:

$\operatorname{Pr}\left(y_{i}=1 \mid x_{i}\right)=\frac{\exp \left(x_{i}^{T} \beta\right)}{1+\exp \left(x_{i}^{T} \beta\right)}$

${ }^{4}$ Sistema Integrado de Transporte del Valle de Aburrá.

Revista de Economía Institucional, vol. 22, n. ${ }^{\circ} 42$, Primer semestre/2020, Pp. $297-323$ ISSN OI 24-5996/E-ISSN 2346-2450 
Para la frecuencia se estima un CRM porque es posible identificar un comportamiento progresivo entre las categorías ordenadas (Hardin et al., 2007; Hou y Archer, 2015). En efecto, las artes escénicas son especiales porque tienen un valor simbólico que se debe descifrar para que su consumo sea satisfactorio. Para esto es necesario que el individuo tenga una dotación de capital humano suficiente para que su experiencia de "apreciación cultural” satisfaga sus necesidades de consumo; este capital es formado por su stock de capital inicial, sus experiencias de consumo anteriores y su inversión en el consumo de otros bienes culturales a lo largo de su vida (Stigler y Becker, 1977; Lévy y Montmarquette, 1996; Ateca, 2009).

Se puede decir entonces que un individuo participa frecuente o asiduamente en artes escénicas porque ha experimentado un proceso de formación del gusto que lo lleva a incrementar la intensidad del consumo. Por tanto, es adecuado estimar un CRM. El CRM define la probabilidad de que el individuo pertenezca a una categoría $c$, condicionada al tránsito por categorías inferiores y al vector de variables explicativas $x_{i}$, como proponen Hou y Archer (2015):

$\delta_{i c}=\operatorname{Pr}\left(y_{i}=c \mid y_{i} \leq c, x_{i}\right)=\frac{\exp \left(\alpha_{c}+x_{i}^{T} \beta\right)}{1+\exp \left(\alpha_{c}+x_{i}^{T} \beta\right)}$

donde $\alpha_{c}$ define los $C-1$ umbrales y $\beta$ es el vector de parámetros a estimar.

La aplicación adecuada de los modelos propuestos requiere la identificación de las variables explicativas relevantes, es decir, las de mayor capacidad para explicar y predecir la participación en artes escénicas y su frecuencia en el caso de Medellín (Gallón y Vásquez, 2014). Idealmente, se espera que el número de variables explicativas $p$ sea menor que el número de observaciones $n$ para obtener resultados parsimoniosos y fáciles de interpretar. Sin embargo, cuando la dimensión del vector de variables regresoras $\mathcal{X}$ crece, la elección de las variables que debe incluir el modelo es un proceso complejo y puede generar problemas de alta dimensionalidad (Hastie et al., 2009; Fan et al., 2014).

En la literatura estadística se proponen métodos para tratar problemas de alta dimensionalidad, entre ellos, los modelos regularizados. Estos imponen penalidades al vector de coeficientes $\beta$ asociado al vector de variables explicativas $\chi$, para reducir su magnitud y su varianza e identificar cuáles $\beta_{j}$ son diferentes de cero, lo que implica que la variable es relevante para explicar y predecir la variable dependiente. Un parámetro de regularización $\lambda \geq 0$ determina cuán estricta es la penalidad: cuanto mayor es $\lambda$ más fuerte es la restricción sobre el 
número de variables que se deben incluir (Hastie et al., 2009 y 2015; Gallón y Vásquez, 2014).

En este caso, el número de variables regresoras es inferior al número de observaciones; pero contar con más de 50 variables explicativas, incluidas las dummies de las variables categóricas, afecta la parsimonia del modelo y dificulta la interpretación. Además, la inclusión de variables altamente relacionadas (como el ingreso, el estrato y el nivel educativo) puede afectar la estabilidad de los modelos estimados.

La función de penalidad $P_{\alpha}(\beta)$ elegida es la elastic net, una combinación lineal convexa de la norma $\ell_{1}$ (lasso) y la norma $\ell_{2}$ (ridge) del vector de parámetros $\beta$. A diferencia de la penalidad ridge, esta selecciona las variables fijando el valor de algunos coeficientes en 0 . Con respecto a la penalidad lasso este método tiene mayor capacidad para tratar variables altamente correlacionadas. Siguiendo a Buhlmann y Van de Geer (2011), la penalidad elastic net está representada por la ecuación (4).

$P_{\alpha}(\beta)=\lambda\left[\frac{1}{2}(1-\alpha) \sum_{j=1}^{p} \beta_{j}^{2}+\alpha \sum_{j=1}^{p}\left|\beta_{j}\right|\right]$

donde $\alpha \in[0,1]$ es un parámetro adicional de afinamiento que indica la importancia de cada penalidad en el problema de optimización. La penalidad se impone al negativo de la función de log verosimilitud del modelo de elección discreta respectivo, y regulariza el proceso de estimación de los coeficientes.

El parámetro $\lambda$ se elige usando el método de validación cruzada, que garantiza la optimalidad de la capacidad de predicción (Hastie et al., 2009). En este caso se eligió aleatoriamente el 80\% de la muestra como datos de entrenamiento para las reglas de clasificación y el $20 \%$ como datos de prueba para evaluar la capacidad predictiva de los modelos (Gallón y Vásquez, 2014). En el modelo de regresión logística binaria se obtuvo dos tipos de valores: $\lambda_{\min }$, que minimiza la devianza del modelo, y $\lambda_{1 s e}$, el valor más grande de $\lambda$ para el cual la devianza del modelo está a un error estándar del valor mínimo (Friedman et al., 2010). En el CRM también se seleccionaron dos valores: $\lambda_{\text {aic }}$, que minimiza el criterio de información AIC, y $\lambda_{b i c}$, que minimiza el criterio de información BIC. Cuanto mayor es el valor de $\lambda$, más fuerte es la penalización y la selección de variables es más rigurosa. El valor del parámetro $\alpha$, por su parte, se fijó en 0,5 para que la ponderación de las penalidades ridge y lasso sean iguales. Los modelos se estiman usando los paquetes glmnet (Friedman et al., 2010) y glmnetcr (Archer et al., 2014), desarrollados en el lenguaje R y disponibles bajo licencia pública. 


\section{RESULTADOS}

\section{ANÁLISIS DESCRIPTIVO}

Los datos de la ECVM2014 indican que el 5,4\% de los jefes de hogar entrevistados asistieron a algún evento de artes escénicas en el año anterior a la encuesta. De estos, el 64,5\% lo hizo esporádicamente, el $14,2 \%$ frecuentemente y el $21,2 \%$ asiduamente.

Mapa 1

Distribución espacial de los equipamientos culturales en Medellín (Porcentaje de participación en artes escénicas por comuna)

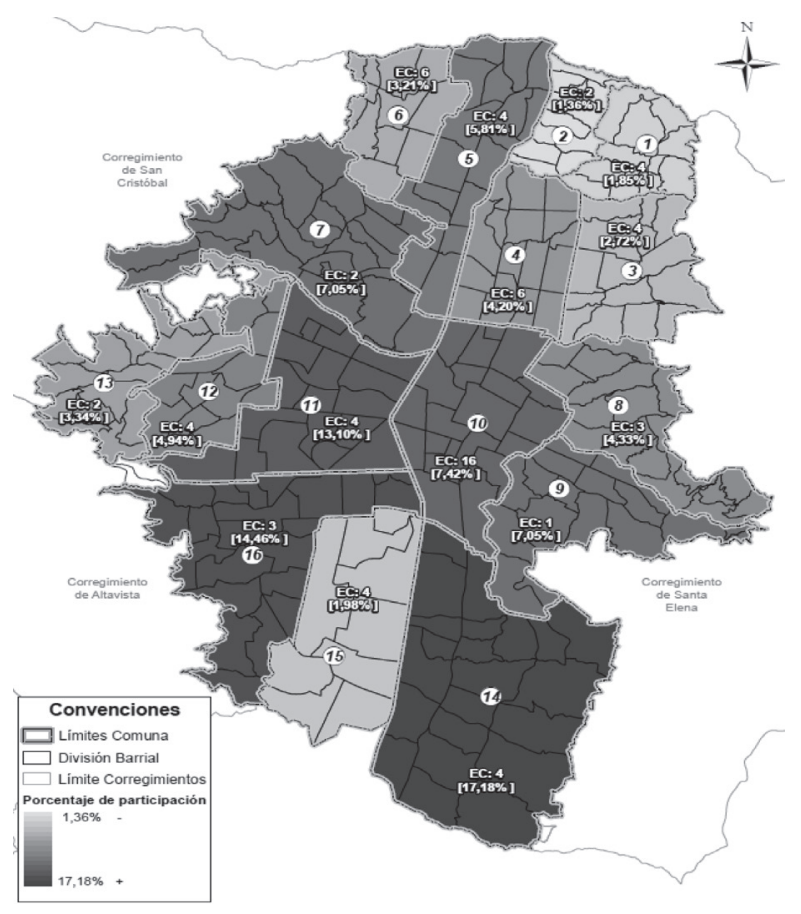

(C): número de comuna. EC: número de equipamientos culturales en la comuna. [ ]: porcentaje de participación en artes escénicas en la comuna.

Fuente: POT2014 y ECVM2014, elaboración propia.

El mapa 1 muestra la distribución espacial de los equipamientos culturales en la ciudad y los porcentajes de participación en artes escénicas por comuna. Las comunas con mayor porcentaje de participación son la 14 (Poblado), 16 (Belén) y 11 (Laureles-Estadio), de estrato socioeconómico alto, medio bajo y medio alto, respectivamente. El mayor número de equipamientos culturales se encuentra en las comunas 10 (La Candelaria), 6 (12 de octubre) y 4 (Aranjuez). De esto se infiere 
que la cercanía a equipamientos culturales no tiene un gran efecto en la asistencia a artes escénicas.

E1 cuadro 1 presenta las características de la muestra completa, asistentes y no asistentes, según las variables explicativas seleccionadas; lo que permite comparar los perfiles de los tres grupos y establecer el efecto preliminar sobre la asistencia. También muestra el valor $\mathrm{p}$ de las pruebas estadísticas, que indica si hay diferencias significativas por variables y grupo de variables entre asistentes y no asistentes. Para las variables continuas se hace una prueba de diferencia de medias y para las cualitativas, una prueba chi cuadrado de Pearson. Como resultado, se evidencian diferencias significativas en el sexo, estado civil, ingreso, estrato, nivel educativo, educación en artes, actividades deportivas, educación, número de personas en el hogar, comuna y cercanía al metro. El análisis de la concentración de los asistentes en las categorías de las variables independientes corrobora este resultado.

Cuadro 1

Características de los participantes en artes escénicas (Porcentaje)

\begin{tabular}{|c|c|c|c|c|c|c|}
\hline & Variable & Categoría & $\begin{array}{l}\text { Muestra } \\
\text { completa }\end{array}$ & Asistentes & $\begin{array}{l}\text { No } \\
\text { asistentes }\end{array}$ & Valor $\mathrm{p}$ \\
\hline \multirow{16}{*}{ 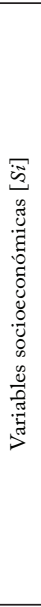 } & Edad $^{a} /$ & Edad & 52,8 & 52,9 & 52,8 & 0,865 \\
\hline & \multirow{2}{*}{ Sexo } & Mujer & 50,5 & 46,2 & 50,7 & \multirow{2}{*}{0,027} \\
\hline & & Hombre & 49,5 & 53,8 & 49,3 & \\
\hline & Minoría & $\begin{array}{l}\text { Pertenece a una minoría étnica (indí- } \\
\text { gena, negro, raizal, rom, gitano) }\end{array}$ & 4,98 & 4,09 & 5,03 & 0,311 \\
\hline & \multirow{6}{*}{ Estado civil } & Soltero & 22,16 & 27,57 & 21,84 & \multirow{6}{*}{0,000} \\
\hline & & Casado & 36,26 & 36,81 & 36,23 & \\
\hline & & Viudo & 12,71 & 10,15 & 12,86 & \\
\hline & & Separado o divorciado & 10,53 & 15,15 & 10,26 & \\
\hline & & $\begin{array}{l}\text { No está casado y vive en pareja hace } \\
\text { menos de dos años }\end{array}$ & 2,26 & 1,67 & 2,30 & \\
\hline & & $\begin{array}{l}\text { No está casado y vive en pareja hace } \\
\text { dos años o mas }\end{array}$ & 16,07 & 8,63 & 16,50 & \\
\hline & \multirow{6}{*}{ Ingreso } & $\$ 0-\$ 700.000$ & 34,51 & 17,57 & 35,48 & \multirow{6}{*}{0,000} \\
\hline & & $\$ 700.000-\$ 1.400 .000$ & 41,21 & 37,87 & 41,40 & \\
\hline & & $\$ 1.400 .000-\$ 2.100 .000$ & 13,43 & 20,60 & 13,02 & \\
\hline & & $\$ 2.100 .000-\$ 2.800 .000$ & 4,68 & 7,72 & 4,51 & \\
\hline & & $\$ 2.800 .000-\$ 4.200 .000$ & 3,78 & 9,09 & 3,47 & \\
\hline & & Más de $\$ 4.200 .000$ & 2,38 & 7,12 & 2,11 & \\
\hline \multirow{6}{*}{\multicolumn{2}{|c|}{ Estrato }} & Bajo bajo (1) & 12,71 & 5,30 & 13,13 & \multirow{6}{*}{0,000} \\
\hline & & Bajo (2) & 32,56 & 13,79 & 33,64 & \\
\hline & & Medio bajo (3) & 29,61 & 28,78 & 29,66 & \\
\hline & & Medio (4) & 11,79 & 18,33 & 11,42 & \\
\hline & & Medio alto (5) & 8,64 & 20,00 & 7,99 & \\
\hline & & Alto (6) & 4,68 & 13,79 & 4,16 & \\
\hline
\end{tabular}




\begin{tabular}{|c|c|c|c|c|c|c|}
\hline & Variable & Categoría & $\begin{array}{l}\text { Muestra } \\
\text { completa }\end{array}$ & Asistentes & $\begin{array}{c}\text { No } \\
\text { asistentes }\end{array}$ & Valor $\mathrm{p}$ \\
\hline \multirow{10}{*}{ 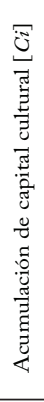 } & \multirow{7}{*}{ Nivel educativo } & Ninguno & 15,74 & 5,15 & 16,35 & \multirow{7}{*}{0,000} \\
\hline & & Primaria & 29,01 & 12,72 & 29,94 & \\
\hline & & Secundaria & 6,97 & 2,87 & 7,20 & \\
\hline & & Media & 25,22 & 25,75 & 25,19 & \\
\hline & & Técnico / Tecnológico & 9,43 & 14,54 & 9,14 & \\
\hline & & Universidad & 9,80 & 24,84 & 8,94 & \\
\hline & & Posgrado & 3,82 & 14,09 & 3,23 & \\
\hline & $\begin{array}{l}\text { Educación } \\
\text { específica }\end{array}$ & $\begin{array}{l}\text { Educación en bellas artes, humanida- } \\
\text { des y/o ciencias religiosas }\end{array}$ & 0,66 & 2,27 & 0,57 & 0,000 \\
\hline & $\begin{array}{l}\text { Equipamientos } \\
\text { culturales }\end{array}$ & $\begin{array}{l}\text { Número de equipamientos culturales } \\
\text { en el barrio }\end{array}$ & 0,368 & 0,391 & 0,367 & 0,454 \\
\hline & $\begin{array}{l}\text { Actividades } \\
\text { deportivas }\end{array}$ & Realiza actividades deportivas & 17,55 & 44,24 & 16,03 & 0,000 \\
\hline \multirow{9}{*}{ 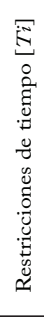 } & \multirow{8}{*}{ Ocupación } & Trabajador & 49,98 & 51,06 & 49,92 & \multirow{8}{*}{0,000} \\
\hline & & Busca trabajo & 3,06 & 2,27 & 3,10 & \\
\hline & & Estudiante & 1,32 & 3,94 & 1,17 & \\
\hline & & Oficios del Hogar & 23,23 & 15,60 & 23,67 & \\
\hline & & Rentista & 0,21 & 0,60 & 0,19 & \\
\hline & & Jubilado o pensionado & 16,74 & 23,48 & 16,36 & \\
\hline & & Otra actividad & 4,22 & 2,72 & 4,31 & \\
\hline & & Incapacitado & 1,22 & 0,30 & 1,27 & \\
\hline & $\begin{array}{l}\text { Personas en el } \\
\text { hogar }\end{array}$ & Número & 3,52 & 2,91 & 3,55 & 0,000 \\
\hline \multirow{17}{*}{ 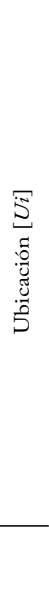 } & \multirow{16}{*}{ Comuna } & Comuna 1 & 5,62 & 1,81 & 5,84 & \multirow{16}{*}{0,000} \\
\hline & & Comuna 2 & 4,66 & 1,36 & 4,84 & \\
\hline & & Comuna 3 & 7,15 & 1,51 & 7,48 & \\
\hline & & Comuna 4 & 6,38 & 4,35 & 6,49 & \\
\hline & & Comuna 5 & 5,54 & 5,45 & 5,54 & \\
\hline & & Comuna 6 & 7,22 & 2,72 & 7,48 & \\
\hline & & Comuna 7 & 7,75 & 6,36 & 7,82 & \\
\hline & & Comuna 8 & 6,80 & 4,70 & 6,91 & \\
\hline & & Comuna 9 & 6,90 & 6,67 & 6,91 & \\
\hline & & Comuna 10 & 4,47 & 7,88 & 4,27 & \\
\hline & & Comuna 11 & 6,04 & 13,78 & 5,60 & \\
\hline & & Comuna 12 & 5,10 & 5,45 & 5,08 & \\
\hline & & Comuna 13 & 7,27 & 3,63 & 7,48 & \\
\hline & & Comuna 14 & 6,30 & 18,48 & 5,61 & \\
\hline & & Comuna 15 & 3,03 & 1,96 & 3,09 & \\
\hline & & Comuna 16 & 9,73 & 13,78 & 9,50 & \\
\hline & Transporte & $\begin{array}{l}\text { El centroide del barrio de residencia } \\
\text { está dentro del buffer de alguna de las } \\
\text { estaciones del Sistema Metro }\end{array}$ & 51,18 & 47,12 & 51,51 & 0,0351 \\
\hline
\end{tabular}

a/ Esta variable es continua, por tanto, se reporta la media en cada una de las muestras.

Fuente: ECVM2014, elaboración propia.

El análisis verifica la multicolinealidad entre estrato, ingreso y nivel educativo, pues la educación determina el tipo de empleo, que se refleja en mejores salarios y la posibilidad de vivir en zonas de estrato más alto. Para ello, se hacen las pruebas chi cuadrado de Pearson que se muestran en el cuadro 2 , donde se observan las relaciones de dependencia entre cada par de variables sospechosas de generar multicolinealidad. 
Cuadro 2

Dependencia entre las variables explicativas

\begin{tabular}{llccc}
\hline Variables & & Nivel educativo & Estrato & Nivel de ingresos \\
\hline \multirow{3}{*}{ Nivel educativo } & Valor $\mathrm{p}$ & 0,000 & - & - \\
\cline { 2 - 5 } & Coeficiente de contingencia & 1,000 & - & - \\
\cline { 2 - 5 } & Coeficiente de correlación & 1,000 & - & - \\
\hline \multirow{3}{*}{ Estrato } & Valor $\mathrm{p}$ & 0,000 & 0,000 & - \\
\cline { 2 - 5 } & Coeficiente de contingencia & 0,505 & 1,000 & - \\
\cline { 2 - 5 } & Coeficiente de correlación & 0,513 & 1,000 & - \\
\hline \multirow{3}{*}{ Nivel de ingreso } & Valor $\mathrm{p}$ & 0,0000 & 0,000 & 0,000 \\
\cline { 2 - 5 } & Coeficiente de contingencia & 0,468 & 0,583 & 1,000 \\
\cline { 2 - 5 } & Coeficiente de correlación & 0,459 & 0,614 & 1,000 \\
\hline
\end{tabular}

Fuente: ECVM2014, elaboración propia.

Para determinar el grado de asociación entre ellas se calculan los coeficientes de contingencia y de correlación, que indican relaciones de dependencia moderadas a altas, pues en todos los casos sus valores son superiores a 0,45 . Una opción para evitar los problemas asociados a la multicolinealidad, como la pérdida de precisión de los estimadores, es eliminar alguna de las variables correlacionadas y comparar la sensibilidad de los resultados, como hacen Muñiz et al. (2017). Con este procedimiento se corre el riesgo de incurrir en el sesgo de variables omitidas. E1 enfoque de la regularización de modelos de elección discreta que se adopta aquí permite incluir todas las variables explicativas (correlacionadas o no); el método selecciona las más relevantes para explicar la variable dependiente respectiva y corregir las imprecisiones causadas por la multicolinealidad, sin eliminar la información de una de las variables.

\section{LA DECISIÓN DE ASISTIR}

La gráfica 1 muestran los resultados del proceso de selección de variables en el modelo logístico regularizado para la decisión de asistir a artes escénicas. El número de variables seleccionadas con el parámetro $\lambda_{\min }$ (46) es mayor que el número seleccionado con $\lambda_{1 s e}(12)$, aunque el costo por mejorar la parsimonia es el aumento del error de devianza en una desviación estándar, es decir, el modelo pierde capacidad explicativa. Además, el error de clasificación del modelo en los datos de prueba crece del 30,5\% al 32,3\% cuando se pasa de $\lambda_{\min }$ a $\lambda_{1 s e^{*}}$ Aunque la capacidad de clasificación del modelo es baja, aquí se utilizan los resultados con fines de interpretación. El cuadro 3 reporta las estimaciones de los coeficientes para los dos valores óptimos del parámetro de regularización, y los efectos marginales. Las variables 
con un coeficiente estimado igual a 0 no son relevantes para explicar la variable dependiente.

Los resultados para el parámetro de regularización indican que la decisión de participar en artes escénicas está determinada principalmente por la alta dotación de capital cultural, medida por la educación formal, la educación en artes y la asistencia a actividades deportivas. Con respecto a los individuos sin educación, las personas con un nivel educativo mayor que el de secundaria tienen mayor probabilidad de participación que las de niveles inferiores. E, igual que en los trabajos de Ateca (2009), Aguado y Palma (2012) y Muñiz et al. (2017), se encuentra un efecto monótonamente creciente del nivel educativo, reflejado en el aumento del efecto marginal a medida que se avanza a niveles superiores; el mayor efecto marginal es el de posgrado $(7,4 \%)$. La educación específica en artes y humanidades tiene el efecto positivo esperado: la probabilidad de asistencia de personas con título universitario en estas áreas es un 3,9\% mayor.

\section{Gráfica 1}

Ejercicio de selección de variables en el modelo logístico binario
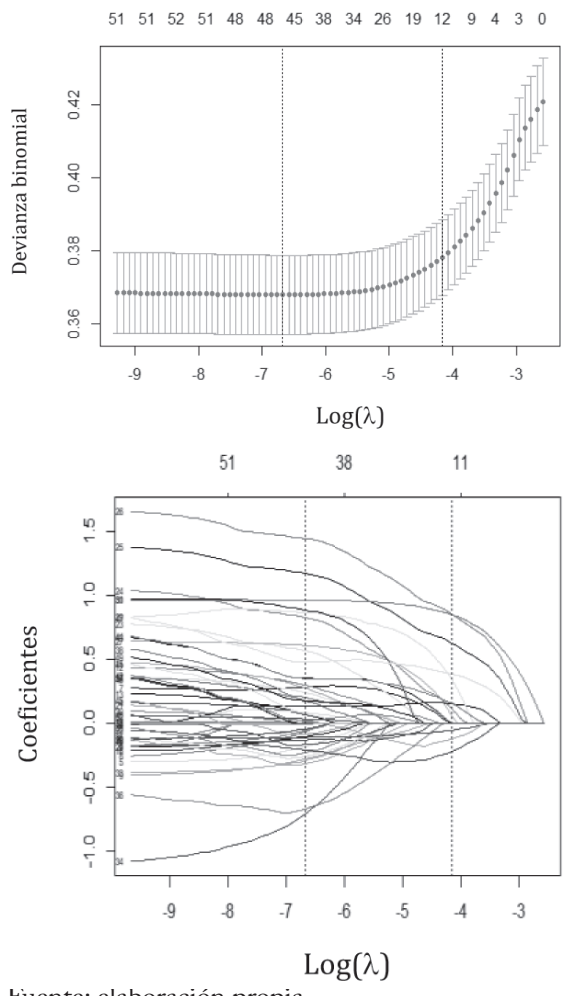

Fuente: elaboracion propia. 
Cuadro 3

Resultados de los modelos para la asistencia a artes escénicas

\begin{tabular}{|c|c|c|c|c|c|c|}
\hline & \multirow[b]{2}{*}{ Variable } & \multirow[b]{2}{*}{$\begin{array}{l}\text { Categoría } \\
\text { Coeficiente }\end{array}$} & \multicolumn{2}{|c|}{$\lambda_{\min }$} & \multicolumn{2}{|c|}{$\lambda_{1 s e}$} \\
\hline & & & $\begin{array}{c}\text { Efectos } \\
\text { marginales }\end{array}$ & Coeficiente & $\begin{array}{c}\text { Efectos } \\
\text { marginales }\end{array}$ & \\
\hline \multirow{20}{*}{ 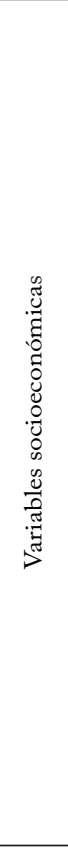 } & Intercepto & Intercepto & $-3,5832$ & $-0,1834$ & $-3,0268$ & $-0,1549$ \\
\hline & \multirow{2}{*}{ Edad } & Edad & & & - & - \\
\hline & & Edad2 & 0,0000 & 0,0000 & - & - \\
\hline & $\begin{array}{l}\text { Sexo } \\
\text { (Mujer) }\end{array}$ & Hombre & $-0,1054$ & $-0,0054$ & - & - \\
\hline & $\begin{array}{l}\text { Minoría } \\
\text { (No pertenece } \\
\text { a una minoría) } \\
\end{array}$ & $\begin{array}{l}\text { Pertenece a una } \\
\text { minoría }\end{array}$ & $-0,0697$ & 0,0036 & - & - \\
\hline & \multirow{5}{*}{$\begin{array}{l}\text { Estado civil } \\
\text { (Soltero) }\end{array}$} & Casado & $-0,1838$ & $-0,0094$ & - & - \\
\hline & & Viudo & $-0,0622$ & $-0,0032$ & $\begin{array}{ll}- \\
\end{array}$ & 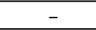 \\
\hline & & Separado o divorciado & 0,2810 & 0,0144 & - & - \\
\hline & & $\begin{array}{c}\text { No está casado y vive } \\
\text { en pareja hace menos } \\
\text { de dos años }\end{array}$ & $-0,0674$ & $-0,0034$ & - & - \\
\hline & & $\begin{array}{c}\text { No está casado y vive } \\
\text { en pareja hace dos } \\
\text { años o mas }\end{array}$ & $-0,2846$ & $-0,0146$ & - & - \\
\hline & \multirow{5}{*}{$\begin{array}{l}\text { Ingreso } \\
(\$ 0-\$ 700.000)\end{array}$} & $\$ 700.000-\$ 1.400 .000$ & 0,2376 & 0,0122 & - & - \\
\hline & & $\begin{array}{c}\$ 1.400 .001- \\
\$ 2.100 .000\end{array}$ & 0,2984 & 0,0153 & - & - \\
\hline & & $\begin{array}{l}\$ 2.100 .001- \\
\$ 2.800 .000\end{array}$ & 0,0768 & 0,0039 & - & - \\
\hline & & $\begin{array}{c}\$ 2.800 .000- \\
\$ 4.200 .000\end{array}$ & 0,1767 & 0,0090 & - & - \\
\hline & & Más de $\$ 4.200 .000$ & 0,2736 & 0,0140 & - & - \\
\hline & \multirow{5}{*}{$\begin{array}{l}\text { Estrato } \\
(1 \text { - Bajo Bajo })\end{array}$} & $2-$ Bajo & $-0,2088$ & $-0,0107$ & $-0,2214$ & $-0,0113$ \\
\hline & & 3 - Medio bajo & 0,0833 & 0,0043 & - & - \\
\hline & & 4-Medio & - & - & - & - \\
\hline & & 5 - Medio alto & 0,1339 & 0,0069 & 0,1504 & 0,0077 \\
\hline & & $6-$ Alto & - & - & & \\
\hline \multirow{9}{*}{ 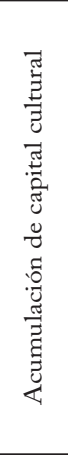 } & \multirow{6}{*}{$\begin{array}{l}\text { Nivel educativo } \\
\text { (Ninguno) }\end{array}$} & Primaria & - & - & $-0,1241$ & $-0,0064$ \\
\hline & & Secundaria & $-0,1320$ & $-0,0068$ & - & - \\
\hline & & Media & 0,5654 & 0,0289 & - & - \\
\hline & & Técnico /tecnológico & 0,8263 & 0,0423 & 0,1141 & 0,0058 \\
\hline & & Universidad & 1,1750 & 0,0601 & 0,6279 & 0,0321 \\
\hline & & Posgrado & 1,4477 & 0,0741 & 0,8589 & 0,0440 \\
\hline & $\begin{array}{l}\text { Educación } \\
\text { específica } \\
\text { (No tiene } \\
\text { educación en } \\
\text { artes) } \\
\end{array}$ & Educación en artes & 0,6093 & 0,0312 & 0,0561 & 0,0029 \\
\hline & $\begin{array}{l}\text { Actividades } \\
\text { deportivas } \\
\text { (No participa) }\end{array}$ & Actividades deportivas & 0,9654 & 0,0494 & 0,8493 & 0,0435 \\
\hline & $\begin{array}{l}\text { Equipamientos } \\
\text { culturales }\end{array}$ & $\begin{array}{l}\text { Equipamientos } \\
\text { culturales }\end{array}$ & 0,0323 & 0,0017 & & \\
\hline \multirow{8}{*}{ 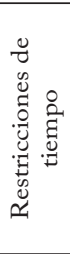 } & \multirow{7}{*}{$\begin{array}{l}\text { Ocupación } \\
\text { (Trabaja) }\end{array}$} & Buscando trabajo & $-0,0453$ & $-0,0023$ & & \\
\hline & & Estudiando & 0,8683 & 0,0445 & 0,3417 & 0,0175 \\
\hline & & Oficios del hogar & & & & \\
\hline & & Rentista & 0,8886 & 0,0455 & & \\
\hline & & Jubilado o pensionado & 0,0174 & 0,0009 & & \\
\hline & & Otra actividad & $-0,2948$ & $-0,0151$ & & \\
\hline & & Incapacitado & $-0,7118$ & $-0,0364$ & & \\
\hline & $\begin{array}{l}\text { Personas en el } \\
\text { hogar }\end{array}$ & Personas en el hogar & $-0,1124$ & $-0,0058$ & $-0,0562$ & $-0,0029$ \\
\hline
\end{tabular}




\begin{tabular}{|c|c|c|c|c|c|c|}
\hline & \multirow[b]{2}{*}{ Variable } & \multirow{2}{*}{$\begin{array}{l}\text { Categoría } \\
\text { Coeficiente }\end{array}$} & \multicolumn{2}{|c|}{$\lambda_{\min }$} & \multicolumn{2}{|c|}{$\lambda_{1 s e}$} \\
\hline & & & $\begin{array}{c}\text { Efectos } \\
\text { marginales }\end{array}$ & Coeficiente & $\begin{array}{c}\text { Efectos } \\
\text { marginales }\end{array}$ & \\
\hline \multirow{16}{*}{ 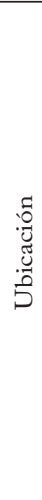 } & \multirow{15}{*}{$\begin{array}{l}\text { Comuna } \\
\text { (Comuna 1) }\end{array}$} & Comuna 2 & $-0,2596$ & $-0,0133$ & & \\
\hline & & Comuna 3 & $-0,6732$ & $-0,0345$ & & \\
\hline & & Comuna 4 & & & & \\
\hline & & Comuna 5 & 0,1994 & 0,0102 & & \\
\hline & & Comuna 6 & $-0,3175$ & $-0,0163$ & & \\
\hline & & Comuna 7 & & & & \\
\hline & & Comuna 8 & 0,0778 & 0,0040 & & \\
\hline & & Comuna 9 & 0,0129 & 0,0007 & & \\
\hline & & Comuna 10 & 0,3527 & 0,0181 & & \\
\hline & & Comuna 11 & 0,3496 & 0,0179 & 0,1502 & 0,0077 \\
\hline & & Comuna 12 & 0,0841 & 0,0043 & & \\
\hline & & Comuna 13 & $-0,1693$ & $-0,0087$ & & \\
\hline & & Comuna 14 & 0,4815 & 0,0247 & 0,3829 & 0,0196 \\
\hline & & Comuna 15 & $-0,2346$ & $-0,0120$ & & \\
\hline & & Comuna 16 & 0,1754 & 0,0090 & & \\
\hline & $\begin{array}{l}\text { Transporte } \\
\text { (No tiene acce- } \\
\text { so a transporte) }\end{array}$ & Transporte & $-0,0081$ & $-0,0004$ & & \\
\hline
\end{tabular}

Los niveles de referencia de las variables cualitativas que se interpretan van entre paréntesis.

Fuente: ECVM2, elaboración propia.

La participación en actividades deportivas, que se considera parte del capital cultural (Curtis et al., 2003; Stempel, 2005), influye positivamente en la participación en artes escénicas. Aunque en teoría ambas son intensivas en tiempo y compiten en la asignación del tiempo de ocio, los resultados muestran que hay una relación de complementariedad: para quienes practican deporte, la probabilidad de asistir a artes escénicas es 4,9\% más alta. Este resultado, similar a los de Hallmann et al. (2017) y Muñiz (2011), obedece a que quienes practican estas actividades valoran más el tiempo de ocio y por ello lo dedican a aquellas que contribuyen al cultivo de la mente y el cuerpo.

Las limitaciones de tiempo son relevantes para explicar las decisiones de asistencia a artes escénicas, como también reportan Machado et al. (2017). Los rentistas y los estudiantes que, en comparación con los trabajadores, disponen de más tiempo para actividades de ocio, tienen mayor probabilidad de asistencia; el efecto marginal de los rentistas es de un 4,6\% y el de los estudiantes de un 4,5\%. Los incapacitados para trabajar tienen un 3,6\% menos probabilidad de participar, lo que se explica por sus restricciones para desplazarse. E1 número de personas en el hogar, proxy de las responsabilidades familiares, reduce la probabilidad de asistencia.

En el caso de las variables socioeconómicas se encontró que el coeficiente de los niveles de ingreso es positivo, es decir, las personas con un ingreso superior a $\$ 700.000$ tienen una mayor probabilidad de asistencia con respecto a quienes tienen un menor ingreso, aunque el efecto marginal en todos los casos es inferior al 2\%. La comparación 
de los efectos marginales indica que el nivel educativo predice mejor la participación cultural que el nivel de ingreso, un resultado que también encuentran Borgonovi (2004), Ateca (2008) y, Fernández y Prieto (2009). En cambio, el estrato socioeconómico no resultó relevante porque los efectos marginales de las categorías con coeficientes diferentes de 0 son inferiores al 1\%. Así, al aislar el efecto de los ingresos y del nivel educativo, el estrato socioeconómico del lugar de residencia explica poco las decisiones de participación cultural.

Residir en la comuna 14 (Poblado) aumenta la probabilidad de asistencia en más del 2\%; y en la 10 (Belén) y en la 11 (Laureles-Estadio) en un 1,8\%; un resultado consistente con el análisis descriptivo preliminar. El efecto positivo de residir en estas zonas obedece más a factores relacionados con sus características sociales, como el mayor nivel de educación cultural o la acumulación de capital social, que, a la dotación cultural o equipamientos de transporte, cuyo efecto se aisló al considerarlas como variables independientes en el modelo. Otros factores -sexo, edad, estado civil o etnia- no tienen efectos relevantes en la asistencia, dado que sus efectos marginales son muy pequeños.

Para precisar los determinantes que más influyen en la decisión de participar se hizo más rigurosa la selección de variables tomando el parámetro de regularización $\lambda_{1 s e}$. El número de variables relevantes se redujo a 12, con el costo de aumentar el error de devianza en una desviación estándar. Los resultados, que se presentan en el cuadro 3, corroboran que el capital cultural es el principal determinante de la asistencia a artes escénicas: los tres niveles educativos más altos, la participación en actividades deportivas y la formación en artes y humanidades siguen siendo relevantes y su efecto sobre la probabilidad de asistencia es positivo; el efecto de la participación en actividades deportivas es similar al del nivel de posgrado. Otros factores que mantienen un efecto positivo son la ocupación, específicamente ser estudiante, y residir en las comunas 11 o 14.

\section{LA FRECUENCIA DE ASISTENCIA}

Con respecto al CRM estimado para la frecuencia de la asistencia a eventos de artes escénicas, la gráfica 2 muestra los resultados del proceso de selección de los parámetros de regularización óptimos que minimizan los criterios de información $\operatorname{AIC}\left(\lambda_{A I C}\right)$ y $\operatorname{BIC}\left(\lambda_{B I C}\right)$. Con el parámetro $\lambda_{A I C}$ se seleccionaron 34 variables relevantes, aunque la trayectoria converge rápidamente a 0 conforme aumenta el valor de $\lambda$, de modo que cuando se considera $\lambda_{B I C}$ solo es relevante una variable. E1 cuadro 4 presenta los valores de los coeficientes de las variables 
relevantes para cada parámetro de penalización considerado. Las estimaciones solo incluyen la muestra de participantes.

Usando el valor de penalización $\lambda_{A I C}$, los resultados muestran que la frecuencia de asistencia a artes escénicas está determinada principalmente por el nivel de ingreso, las restricciones de tiempo, la formación en artes y humanidades, la participación en prácticas deportivas y la comuna de residencia. En particular, los asistentes con niveles de ingreso de $\$ 1.400 .000$ a $\$ 4.200 .000$ tienen más probabilidad de asistir con más frecuencia que quienes ganan menos de $\$ 700.000$. Esto indica que las barreras de ingreso son importantes para determinar la intensidad con la que se participa en los eventos de artes escénicas en Medellín.

En cuanto a la importancia del capital cultural, los individuos con formación en artes y humanidades y quienes participan en actividades deportivas tienen mayor probabilidad de pertenecer al grupo de mayor frecuencia de asistencia. Además, la educación universitaria influye de manera positiva y significativa en la mayor asistencia. Este resultado es coherente con Ateca (2008), quien encontró que la educación aumenta la probabilidad de asistencia a eventos culturales, pero solamente la educación en los niveles más altos aumenta la frecuencia.

Gráfica 2

Ejercicio de selección de variables en modelo ordenado

AIC

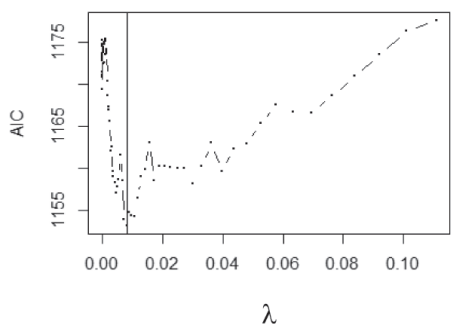

BIC

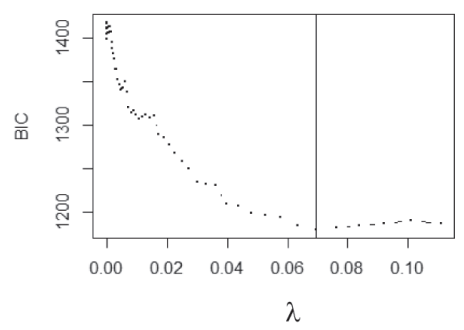

Trayectoria del coeficiente

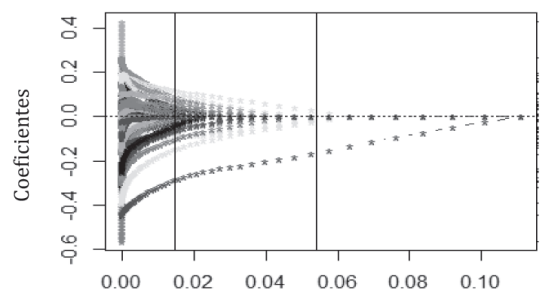

$\lambda$

Fuente: elaboración propia. 
Cuadro 4

Estimación puntual del CRM

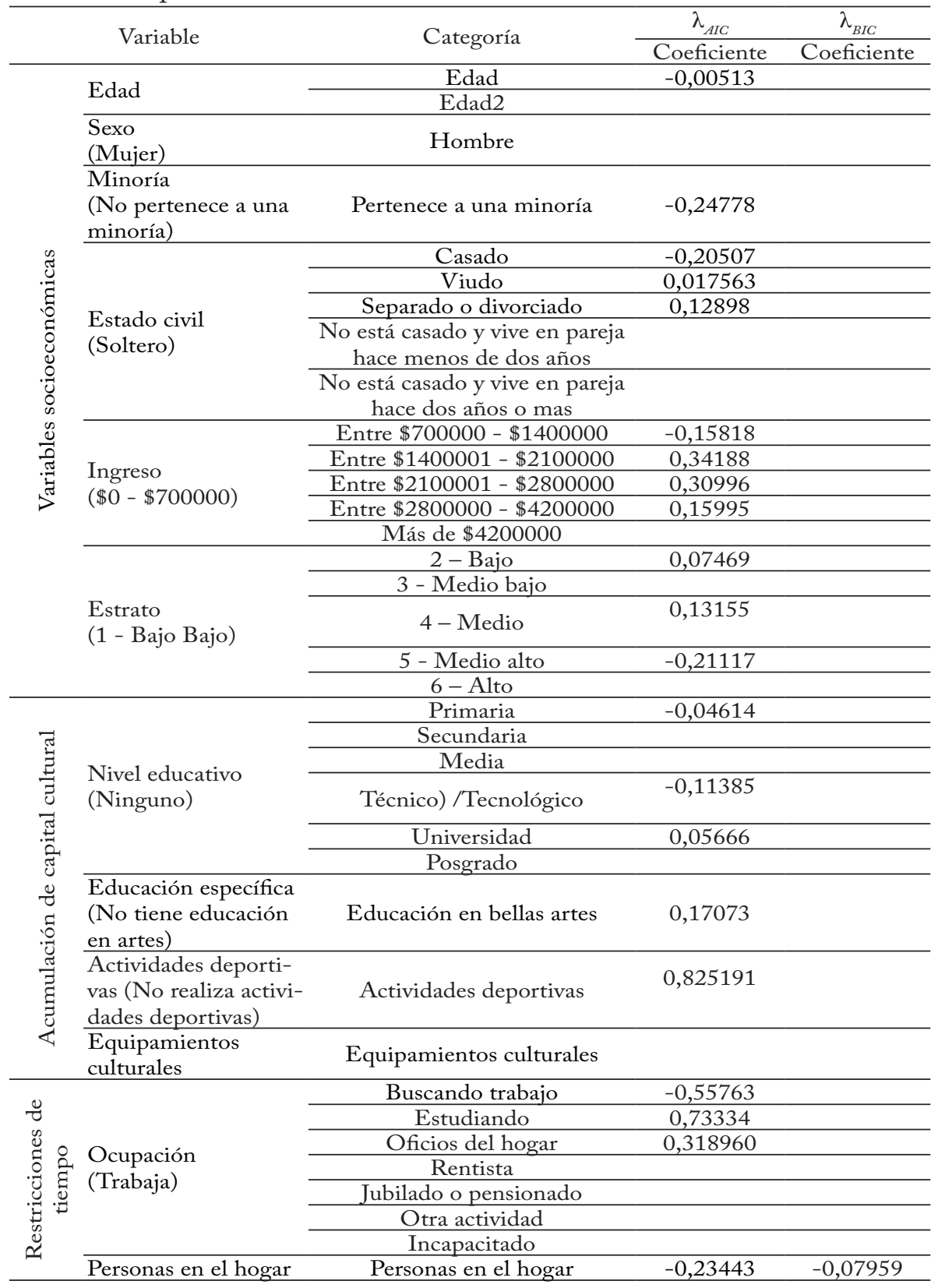




\begin{tabular}{|c|c|c|c|c|}
\hline \multirow{2}{*}{\multicolumn{2}{|c|}{ Variable }} & Categoría & $\lambda_{A I C}$ & $\lambda_{B I C}$ \\
\hline & & Coeficiente & Coeficiente & Coeficiente \\
\hline \multirow{18}{*}{ 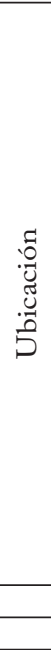 } & \multirow{15}{*}{$\begin{array}{l}\text { Comuna } \\
\text { (Comuna 1) }\end{array}$} & Comuna 2 & & \\
\hline & & Comuna 3 & 0,49859 & \\
\hline & & Comuna 4 & 0,21705 & \\
\hline & & Comuna 5 & $-0,62356$ & \\
\hline & & Comuna 6 & $-0,07601$ & \\
\hline & & Comuna 7 & & \\
\hline & & Comuna 8 & $-0,91930$ & \\
\hline & & Comuna 9 & $-0,41990$ & \\
\hline & & Comuna 10 & $-0,35440$ & \\
\hline & & Comuna 11 & 0,11327 & \\
\hline & & Comuna 12 & 0,18565 & \\
\hline & & Comuna 13 & $-0,55197$ & \\
\hline & & Comuna 14 & 0,24690 & \\
\hline & & Comuna 15 & & \\
\hline & & Comuna 16 & $-0,24224$ & \\
\hline & $\begin{array}{l}\text { Transporte } \\
\text { (No tiene acceso a } \\
\text { transporte) }\end{array}$ & Transporte & 0,19312 & \\
\hline & & & $-0,12448$ & $-0,19015$ \\
\hline & & & 0,0000 & 0,0000 \\
\hline
\end{tabular}

Fuente: ECVM2014, elaboración propia.

Las restricciones de tiempo también resultaron relevantes en el CRM. Este resultado es un reflejo del costo de oportunidad en que incurren los trabajadores, las personas casadas y quienes tienen familias más numerosas por asistir a presentaciones de artes escénicas. Residir en las comunas 3, 4, 11, 12 y 14 incide positivamente en la frecuencia de participación. Cabe señalar que el número de personas en el hogar es el principal determinante de la frecuencia de participación en $\mathrm{Me}$ dellín, debido a que es la única variable con un coeficiente diferente de 0 cuando se considera el parámetro $\lambda_{B I C}$.

\section{CONCLUSIONES}

Este trabajo analiza los determinantes de la participación, y su frecuencia, en artes escénicas en vivo en Medellín utilizando la ECVM2014, un ejercicio que explora los hábitos de consumo en teatro, danza y música de los hogares. E1 5,4\% de los jefes de familia considerados manifestó que participaba; la mayoría en forma ocasional. Los participantes son principalmente hombres, mestizos que residen en las comunas Poblado, Belén y Laureles-Estadio, de estrato socioeconómico alto, medio bajo y medio alto respectivamente, con un nivel de educación superior al promedio.

Los factores más importantes en la decisión de participar y en su intensidad son: el nivel de educación, las restricciones de tiempo 
y el número de personas en el hogar. Estos resultados concuerdan con los de estudios que encuentran que el nivel de educación -una variable proxy del capital cultural- es el principal determinante de la participación y la frecuencia de participación en estas actividades. E1 efecto de las restricciones de tiempo obedece a que el consumo de artes escénicas es intensivo en tiempo; por tanto, el costo de oportunidad del tiempo de ocio es un determinante de la asistencia.

El efecto del ingreso encontrado, a diferencia de lo que se observó en la literatura revisada, es mínimo. Esto puede ser consecuencia de la oferta cultural gratuita en la ciudad, que hace posible asistir a este tipo de espectáculos sin afectar sensiblemente el presupuesto. Así, el bajo nivel de asistencia y de frecuencia observado obedece más a la dotación de capital cultural, que no motiva lo suficiente para ser espectador, que a restricciones de ingreso. Se debe, entonces, fortalecer las políticas de formación y fidelización de públicos para crear nuevas audiencias a partir de la formación de capital cultural.

Este trabajo complementa en tres aspectos los hallazgos de Gómez y Espinal $(2016)^{5}$, quienes utilizaron los mismos datos, pero con una metodología diferente. Primero, los modelos de elección discreta regularizados permiten obtener resultados más precisos sobre la influencia de los determinantes de las decisiones de consumo cultural; un insumo más certero para los hacedores de política cultural. Segundo, en este trabajo se incluyeron tres variables adicionales: la participación en actividades deportivas y recreativas, que es una forma de capital cultural (Stempel, 2005); el número y ubicación de los equipamientos culturales en las diferentes áreas geográficas de la ciudad, que acercan la oferta pública de artes escénicas; y la cercanía a alguna estación del SITVA, para determinar si favorece el acceso a los espectáculos.

Comparando los resultados con los reportados por Gómez y Espinal (2016), se observa que en todos los casos los coeficientes estimados, los efectos marginales y las relaciones de probabilidad son menores en el modelo logístico regularizado. Por dos razones: primero, la regularización reduce la magnitud de los coeficientes para corregir la sobreestimación y la imprecisión ocasionadas por la inclusión de un alto número de variables explicativas y la presencia de multicolinealidad. Segundo, el efecto del nivel educativo, el estrato socioeconómico y la comuna resultó menor porque el modelo incluye variables adicionales, como los equipamientos culturales, de transporte y prácticas de actividades deportivas. No obstante, ambos

5 Disponible en: https://revistas.unal.edu.co/index.php/ede/article/ view/59863 
trabajos obtienen algunos resultados similares: el efecto positivo y monótonamente creciente del nivel educativo a partir de educación media, el efecto positivo de la mayor disponibilidad de tiempo de estudiantes y rentistas, el efecto negativo del número de personas en el hogar, y el efecto positivo de residir en las comunas 14, 10, 11 y 5 .

Los resultados de esta investigación son de especial interés para todas aquellas personas tanto de la industria cultural como los hacedores de la política pública que puedan estar interesados en conocer el perfil de los consumidores, para construir estrategias de fidelización de públicos y garantizar que las experiencias de consumo cultural tengan efectos positivos sobre el bienestar. A pesar de la inversión pública en dispositivos culturales subsidiados, el número de equipamientos culturales en las comunas no resulta un determinante importante en la participación. Esto puede estar influenciado porque los equipamientos culturales incluidos en el POT2014 tomados en esta investigación no tienen en cuenta parques o espacios al aire libre en los que se realiza gran cantidad de eventos de música en la ciudad, actividad a la que manifestaron participar la mayor parte de los entrevistados. Por ejemplo, en el año 2014 participaron cerca de 19.000 jóvenes en el Festival Altavoz, realizado en las instalaciones de la Cancha Cincuentenario, un espacio que no es un equipamiento cultural, por lo cual esta participación no se refleja en los resultados de esta investigación.

Los resultados de este trabajo tienen algunas limitaciones debido a los datos disponibles. Primero, no se dispone de datos de precios de acceso a artes escénicas, lo que impide explicar el efecto estimado del ingreso en la participación y su frecuencia, una variable relevante en todos los estudios revisados. Segundo, no se dispone de información sobre el conocimiento de la población respecto a la oferta cultural; así, se intuye que la baja participación no necesariamente equivale a falta de interés.

Es necesario hacer un mapeo de los escenarios públicos y privados que of recen espectáculos de artes escénicas y estudiar sus estrategias de divulgación; así se podrán complementar los resultados y precisar los efectos de las características de la oferta en la decisión de asistir. También es necesario entender la decisión de no participar de cerca del 94\% de los entrevistados, para identificar otras características del entorno que fomentan la participación, diferentes de las que identifica la literatura. La disponibilidad de datos sobre estos aspectos permitiría mejorar la especificación del modelo y evitar el posible problema de variables omitidas, que pueden limitar el uso de los resultados para 
diseñar políticas públicas y tomar decisiones sobre cómo consolidar una oferta cultural que interese a la audiencia y la disponibilidad a pagar por ella.

\section{REFERENCIAS BIBLIOGRÁFICAS}

Aguado, L. y Palma, L. (2012). Determinants of performing arts participation in Colombia. Conference paper 17th International Conference on Cultural Economics by the ACEI. Kyoto.

Archer, K., Hou, J. et al. (2014). Ordinalgmifs: An R package for ordinal regression in high-dimensional data settings. Cancer informatics, 13, 187-195.

Ateca-A., V. (2008). Determining heterogeneous behavior for theater attendance. Journal of Cultural Economics, 32(2), 127-151.

Ateca-A., V. (2009). El capital humano como determinante del consumo cultural. Estudios de Economía Aplicada, 27(1), 87-110.

Alcaldía de Medellín. (2011). Plan de Desarrollo Cultural 2011-2020, [https://bibliotecasmedellin.gov.co/content/uploads/2015/07/Plan_de_ Desarrollo_Cultural_de_Medellin_2011-2020.pdf].

Alcaldía de Medellín. (2018). Resolución No. 201850021761. Apertura de la Convocatoria de estímulos para el arte y la cultura Fase II, Secretaría de Cultura Ciudadana, [convocatoriasculturamedellin. $\mathrm{com} / \mathrm{sitio} / \mathrm{wp}$-content/uploads/2018/03/RESOLUCIÓN-DE-APERTURA$\mathrm{N}^{\circ}$ 201850021761-FASE-II-2018.pdf].

Baumol, W. y Bowen, W. (1966). Performing arts. The economic dilemma. Cambridge: Twentieth Century Fox.

Becker, G. (1965). A theory of the allocation of time. Economic Journal, 75(29), 493-517.

Becker, G. y Murphy, K. (1988). A theory of rational addiction. Journal of Political Economy, 96(4), 675-700.

Belfiore, E. y Bennett, O. (2007) Rethinking the social impacts of the arts. International Journal of Cultural Policy, 13(2), 135-151.

Bourdieu, P. (1984). Distinction: A social critique of the judgment of taste. Londres: Routledge y Kegan Paul.

Bordieu, P. y Passeron, J. (1996). La reproducción. Elementos para una teoría del sistema de enseñanza, 2. ${ }^{a}$ ed. México DF: Laia.

Borgonovi, F. (2004). Performing arts attendance: an economic approach. Applied Economics, 36, 1871-1885.

Brito, P. y Barros, C. (2005). Learning-by-consuming and the dynamics of the demand and prices of cultural goods. Journal of cultural economics, 29(2), 83-106.

Bühlmann, P. y Van De Geer, S. (2011). Statistics for high-dimensional data: Methods, theory and applications. Heidelberg: Springer.

Cameron, A. y Trivedi, P. (2005). Microeconometrics: Methods and applications. Cambridge: Cambridge University Press.

Castiglione, C. e Infante, D. (2016). Rational addiction and cultural goods: the case of the Italian theatregoer. Journal of Cultural Economics, 4O(2), 163-190. 
Cuenca, M. (2006). Aproximación multidisciplinar a los estudios de ocio. Bilbao: Universidad de Deusto.

Curtis, J., McTeer, W. et al. (2003) Do high school athletes earn more pay? Youth sport participation and earnings as an adult. Sociology of Sport Journal, 20(1), 60-76.

Fan, J., Han, F. et al. (2014). Challenges of big data analysis. National Science Review, 1(2), 293-314.

Fernández B., V. y Prieto R., J. (2009). Análisis de los hábitos de lectura como una decisión económica. Estudios de Economía Aplicada, 27(1), $87-110$.

Frey, B. (1994). Art: The economic point of view. En I. Rizzo y A. Peacock (eds.), Cultural economics and cultural policies (pp. 3-16). Dordrecht: Kluwer.

Friedman, J., Hastie, T. et al. (2010). Regularization paths for generalized linear models via coordinate descent. Journal of Statistical Software, 33(1), 1-22.

Gallón, S. y Vasquez, J. (2016). Aplicación de la teoría de clasificación al problema del abandono estudiantil: Un estudio de caso, [https:// revistas.utp.ac.pa/index.php/clabes/article/view/1019].

Gómez, L. y Espinal, N. (2016). Determinantes de la participación en artes escénicas en Medellín, Colombia. Ensayos de Economía, 26(48), 189-207.

Hallmann, K. et al. (2017). Leisure participation: Modeling the decision to engage in sports and culture. Journal of Cultural Economics, 41(4), 467-487.

Hardin, J., Hilbe, J. y Hilbe, J. (2007). Generalized linear models and extensions. Stata Press.

Hastie, T., Tibshirani, R. y Friedman, J. (2009). The elements of statistical learning: Data mining, inference, and prediction, $5^{\text {th }}$ ed. Nueva York: Springer,

Hastie, T., Tibshirani, R., y Wainwright, M. (2015). Statistical learning with sparsity. Boca Raton: CRC Press.

Horkheimer, M., y Adorno, T. (2009). La industria cultural. Ilustración como engaño de masas. En M. Horkheimer y T. Adorno. Dialéctica de la ilustración. Madrid: Trotta.

Hou, J. y Archer, K. (2015). Regularization method for predicting an ordinal response using longitudinal high-dimensional genomic data. Statistical Applications in Genetics and Molecular Biology, 14(1), 93-111.

Lazcano, I. y Ladabidea, X. (2010). E1 consumo cultural y la creación de audiencias escénicas a través de la participación en eventos escénicos extraordinarios. En: Cuenca et al. (2010). Sobre ocio creativo: situación actual de las ferias de artes escénicas (pp. 49-71). Bilbao: Universidad de Deusto.

Lévy-G., L. y Montmarquette, C. (1996). A microeconometric study of theatre demand. Journal of Cultural Economics, 20(1), 25-50.

Levy-G., L. y Montmarquette, C. (2003). Demand. En Towse, R. (2003). $A$ handbook of cultural economics. Cheltenham: Edward Elgar Publishing.

López Q., A. (1977). Estética de la creatividad. Madrid: Cátedra. 
Machado, A., Golgher, A., Diniz, S. y Gama, L. (2017). Consumption of cultural goods and services and time allocation in Brazil. Nova Economia, 27(1), 35-63.

Matarasso, F. (1997). Use or ornament: The social impact of participation in the arts. Stroud: Comedia.

McCarthy, K. et al. (2001). The performing arts in a new era. Santa Monica: RAND.

Muñiz, C., Rodríguez, P. y Suárez, M. (2011). The allocation of time to sports and cultural activities: An analysis of individual decisions. International Journal of Sport Finance, 6(3), 245-264.

Muñiz, C., Rodríguez, P. y Suárez, M. (2017). Participation in cultural activities: Specification issues. Journal of Cultural Economics, 41(1), 71-93.

Núñez, T.P. (2016). Nuevos desplazamientos en la investigación en cultura: aportes de la Segunda Encuesta Nacional de Participación y Consumo Cultural de Chile. Revista Persona y Sociedad, 26(1), 87-112.

Oliva, C. y Torres, F. (1992). Historia básica del arte escénico. Madrid: Cátedra.

Peterson, R. (1992). Understanding audience segmentation: From elite and mass to omnivore and univore. Poetics, 21, 243-258.

Pnud (2002). Informe de desarrollo humano 2002. Nosotros los chilenos: un desafio cultural. Santiago: Pnud.

Red Cómo Vamos. (2015). Informe de calidad de vida, [redcomovamos. org/wp-content/uploads/2015/02/Bolet\%C3\%ADn6_ICV.RedComoVamos_Febrero2015.pdf].

Rish, E. (2005). El valor de la cultura en los procesos de desarrollo urbano sustentable. Barcelona: Universitat Pompeu Fabra.

Seaman, B. (2006). Empirical studies of demand for the performing arts. En: Ginsburgh, V. y Throsby, D. (2006). Handbook of the economics of art and culture, v. 1 (pp. 415-472). Amsterdam: Elsevier.

Stempel, C. (2005). Adult participation sports as cultural capital. A test of Bourdieu's theory of the field of sports. International Review for the Sociology of Sport, 4O(4), 411-432.

Stigler, G. y Becker, G. (1977). De gustibus non est disputandum. American Economic Review, 67(2), pp. 76-90.

Throsby, D. (2001). Economics and culture. Cambridge: Cambridge University Press.

VV.AA. (2004). Raíces. Culturas tradicionales de España e Iberoamérica. Salamanca: Diputación de Salamanca-IIACYL.

Yeoman, I. (2004). Festival and events management: An international arts and culture perspective. Oxford: Elsevier. 